\title{
Neisseria chenwenguii sp. nov. isolated from the rectal contents of a plateau pika (Ochotona curzoniae)
}

\author{
Gui Zhang $\cdot$ Jing Yang $\cdot$ Xin-He Lai $\cdot$ Shan Lu $\cdot$ Dong Jin $\cdot$ Ji Pu $\cdot$ \\ Xiangning Bai $\cdot$ Xuelian Luo $\cdot$ Yanwen Xiong $\cdot$ Ying Huang $\cdot$ Cuixia Chen • \\ Jianguo Xu
}

Received: 11 September 2018/Accepted: 18 January 2019/Published online: 24 February 2019

(C) The Author(s) 2019

\begin{abstract}
Two Gram-stain negative, catalase positive, coccus shaped bacteria, designated $10023^{\mathrm{T}}$ and 10010 , were isolated from the rectal contents of a plateau pika (Ochotona curzoniae) in Qinghai-Tibet Plateau, China. Based on 16S rRNA gene sequence analysis, phylogenetic trees showed that these two isolates $\left(10023^{\mathrm{T}}, 10010\right)$ group with members of the genus Neisseria. Additionally, these two isolates exhibited high 16S rRNA gene sequence similarity with Neisseria zalophi CSL $7565^{\mathrm{T}}$ (96.98\%), Neisseria wadsworthii WC $05-9715^{\mathrm{T}}(96.92 \%)$ and Neisseria canis ATCC $14687^{\mathrm{T}}(96.79 \%)$. Further phylogenetic
\end{abstract}

Electronic supplementary material The online version of this article (https://doi.org/10.1007/s10482-019-01234-2) contains supplementary material, which is available to authorized users.

G. Zhang $\cdot$ J. Yang $\cdot$ S. Lu $\cdot$ D. Jin $\cdot$ J. Pu .

X. Bai $\cdot$ X. Luo $\cdot$ Y. Xiong $\cdot$ Y. Huang $\cdot$

C. Chen $\cdot$ J. Xu $(\bowtie)$

State Key Laboratory of Infectious Disease Prevention and Control, Collaborative Innovation Center for Diagnosis and Treatment of Infectious Diseases, National Institute for Communicable Disease Control and Prevention, Chinese Center for Disease Control and Prevention, Beijing 102206, People's Republic of China e-mail: xujianguo@icdc.cn

J. Yang $\cdot$ S. Lu $\cdot$ D. Jin $\cdot$ X. Bai $\cdot$ J. Xu Shanghai Institute for Emerging and Re-emerging Infectious Diseases, Shanghai Public Health Clinical Center, Shanghai 201508, People's Republic of China analysis based on the $r p l F$ gene showed that these two novel strains can be easily discriminated from phylogenetically closely related species. Optimal growth was found to occur on BHI agar with 5\% defibrinated sheep blood at $37{ }^{\circ} \mathrm{C}$ and growth was also observed on nutrient agar, Columbia blood agar and chocolate agar plates; however, growth was not observed on MacConkey agar after 7 days. The major cellular fatty acids of these strains were identified as $\mathrm{C}_{16: 0}$ and $\mathrm{C}_{16: 1} \omega 7 \mathrm{c} / \mathrm{C}_{16: 1} \omega 6 \mathrm{c}$. The complete genome size of the type strain $10023^{\mathrm{T}}$ is $2,496,444 \mathrm{bp}$, with DNA G+C content of $54.0 \mathrm{~mol} \%$. The average nucleotide identity values were $73.5-79.3 \%$ between isolate $10023^{\mathrm{T}}$ and reference Neisseria spp. Based on polyphasic analysis, these isolates $\left(10023^{\mathrm{T}}\right.$ and 10010) are considered to represent a novel species in the genus Neisseria, for which the name Neisseria chenwenguii

X.-H. Lai

School of Biology and Food Science, Shangqiu Normal University, Shangqiu 475000, Henan Province, People's Republic of China 
sp. nov. is proposed. The type strain is $10023^{\mathrm{T}}$ $\left(=\operatorname{DSM} 103440^{\mathrm{T}}=\right.$ CGMCC $\left.1.15736^{\mathrm{T}}\right)$.

Keywords Average nucleotide identity - Neisseria . Novel species · Phylogeny

\section{Abbreviation}

ANI Average nucleotide identity

\section{Introduction}

The genus Neisseria is the type genus of the family Neisseriaceae. Members of the genus are non-motile Gram-negative cocci, occurring singly, in pairs, or in short chains, with no flagella (Adeolu and Gupta 2013). There are currently 30 species and 3 subspecies in the genus Neisseria (http://www.Bacterio.net/ Neisseria.html), among which Neisseria meningitidis and Neisseria gonorrhoeae are well-known human pathogens, causing bacterial meningitis and gonorrhoea, respectively (Virji 2009). Other species in the genus Neisseria may be associated with human and animal infections, having been isolated from animals, such as ducks (Murphy et al. 2005), mice (Weyand et al. 2016), sea lions (Volokhov et al. 2018) and clinical specimens or nonviable eggs (Hansen et al. 2017; Wolfgang et al. 2011, 2013; Wroblewski et al. 2017).

The Qinghai-Tibet Plateau, also known as the Third Pole or "the Roof of the World", is at a high altitude and has a high level of ultraviolet radiation, low oxygen and temperature, and hosts wild animal species such as wild pikas (Ochotona curzoniae), snow leopards (Panthera uncia), marmots (Marmota himalayana), Tibetan antelopes (Pantholops hodgsonii) and vultures. Several novel bacterial species have been isolated and characterised from plateau wild animals, including Helicobacter himalayensis (Hu et al. 2015), Escherichia marmotae (Liu et al. 2015), Streptococcus halotolerans (Niu et al. 2016), Streptococcus himalayensis (Niu et al. 2017), Streptococcus pantholopis (Bai et al. 2016), Actinomyces liubingyangii and Actinomyces vulturis (Meng et al. 2017a, b).

The wild plateau pika ( $O$. curzoniae) is a tiny mammal belonging to the Order Lagomorpha, which includes rabbits and hares. The plateau pika is abundant and pika holes can be found all over the grassland, suggesting that the plateau pika functions as a key species on the alpine meadow ecosystem in Qinghai Province, PR China (Hogan 2010). During our research into the microbial diversity in the rectal contents of plateau pika, representatives of a novel species in the genus Neisseria were obtained in 2015. Therefore, in this study, we report the isolation and characterisation of this novel Neisseria species isolated from plateau pika. Two isolates $\left(10023^{\mathrm{T}}, 10010\right)$ were obtained, from different pika samples, which showed around 97\% 16S rRNA gene similarity to several members of the genus Neisseria. In this study, the taxonomic position of these two isolates is reported based on the polyphasic approach, including phylogenetic analysis of genes (16S rRNA and $r p l F$ ), average nucleotide identity (ANI), biochemical and chemotaxonomic characterisation. We conclude that these two isolates are distinct from previously described species of Neisseria and represent a novel species in this genus, for which the name Neisseria chenwenguii sp. nov. is proposed.

\section{Materials and methods}

Isolation, maintenance and cultivation of Neisseria strains

Plateau pikas (O. curzoniae) were identified based on genotype (coxl and cox2, encoding $O$. curzoniae cytochrome c oxidase subunit 1 and 2) and phenotype. They were captured by mouse traps and anaesthetised with ether in Yushu Tibetan Autonomous Prefecture in August 2015, as approved by the ethics committee of the National Institute for Communicable Disease Control and Prevention, China CDC. Colon and rectum samples from hundreds of pikas were collected and aliquoted into $5 \mathrm{~mL}$ sterile tubes containing $2 \mathrm{~mL}$ $30 \%$ (w/v) glycerol buffer. Samples were placed in an icebox before being transported to the local laboratory and stored at $-20{ }^{\circ} \mathrm{C}$, followed by cold chain transportation to deliver the pika samples to our laboratory in Beijing. Isolates (10010 and $10023^{\mathrm{T}}$ ) were obtained from the rectal contents of two separate plateau pika (4266 meters above sea level, $33^{\circ} 01^{\prime} 46^{\prime \prime} \mathrm{N}, 96^{\circ} 47^{\prime} 08^{\prime \prime} \mathrm{E}$, male/adult for $10023^{\mathrm{T}}$, and $4278 \mathrm{~m}, 33^{\circ} 01^{\prime} 58^{\prime \prime} \mathrm{N}, 96^{\circ} 46^{\prime} 57^{\prime \prime} \mathrm{E}$, female/adult for 10010) using BHI agar with $5 \%$ defibrinated sheep 
blood at $37{ }^{\circ} \mathrm{C}, 5 \% \mathrm{CO}_{2}$ for two days. Pure cultures were obtained after three successive transfers of single colonies to the same medium. These two novel isolates were preserved at $-80{ }^{\circ} \mathrm{C}$ as a suspension in Brain Heart Infusion Broth with 30\% (w/v) glycerol.

Neisseria wadsworthii DSM $22247^{\mathrm{T}}$ and Neisseria canis DSM $18000^{\mathrm{T}}$ were obtained from the Leibniz Institute DSMZ-German Collection of Microorganisms and Cell Cultures and cultured under the same conditions as the reference strains.

Phylogenetic analysis

Using the Wizard ${ }^{\circledR}$ Genomic DNA Purification Kit, the total DNA were extracted from isolates 10010 and $10023^{\mathrm{T}}$ cultured on BHI agar with $5 \%$ defibrinated sheep blood under the condition of $37{ }^{\circ} \mathrm{C}, 5 \% \mathrm{CO}_{2}$. Subsequently, the $16 \mathrm{~S}$ rRNA gene was amplified using the forward primer (5'-AGAGTTTGATCCTGGCTCAG-3', corresponding to Escherichia coli positions 8-27) and the reverse primer (5'-ACGGCTACCTTGTTACGACTT-3'; 1492-1512) (Neilan et al. 1997), with these amplification conditions (a single cycle at $95{ }^{\circ} \mathrm{C}$ for $5 \mathrm{~min}$, followed by 30 cycles at $95{ }^{\circ} \mathrm{C}$ for $45 \mathrm{~s}, 54{ }^{\circ} \mathrm{C}$ for $45 \mathrm{~s}$ and $72{ }^{\circ} \mathrm{C}$ for $1.5 \mathrm{~min}$, followed by a final extension at $72{ }^{\circ} \mathrm{C}$ for $10 \mathrm{~min}$ and at $4{ }^{\circ} \mathrm{C}$ hold). A BLAST search based on the EzTaxon database (https://www.ezbiocloud.net/ identify) was performed. The almost complete $16 \mathrm{~S}$ rRNA gene sequences of these two novel strains and the 30 closest taxa identified with validly published names were aligned using the Clustal $\mathrm{W}$ program (Thompson et al. 2002). A phylogenetic tree based on the 16S rRNA gene was constructed by different methods (Neighbour-Joining and Maximum-Likelihood) using the MEGA 7.0 software (www. megasoftware.net) (Kumar et al. 2016).

Phylogenetic analysis of the $r p l F$ gene was also performed as recommended by Bennett et al. $(2013,2014)$ to better discriminate the novel species from its phylogenetically closely related species. $r p l F$ gene sequences of these two novel isolates, Neisseria spp and other related genera were aligned using the Clustal W program, and a Neighbour-Joining tree was constructed with the MEGA 7.0 software (www. megasoftware.net).
Average nucleotide identity and genes similarity analysis

The ANI calculation between a given pair of genomes has been used as the gold standard for microorganism classification, and a value of $95 \%$ is recommended as the threshold for delineating species (Richter and Rossello-Mora 2009). To reaffirm the relationship of the novel species with other species in the genus Neisseria, an ANI calculation was performed using online tools at http://www.ezbiocloud.net/tools.

For the type strain $10023^{\mathrm{T}}$, the complete genome was sequenced using the PacBio sequencing platform (RSII). DNA was initially treated into fragments of appropriate size by g-TUBE. Subsequently, the fragments were damage repaired and ends repaired. Both sides of the DNA fragments were respectively connected with hairpin adapters to creat a dumbbell (set of horse ring) structure, which is known as the SMRTbell. After annealing, the SMRTbell was fixed at the bottom of the ZWM polymerase, for use in the final stage of sequencing. Raw data was assembled by Single Molecule, Real-Time (SMRT) Analysis 2.3.0 (Berlin et al. 2015). The draft genome of isolate 10010 was sequenced using the Illumina PE150 platform and assembled by SOPA denovo. The genomes of these two novel isolates were annotated using the method of best-placed reference protein set and the GeneMarkS+ 4.2 software.

Genes with greater nucleotide sequence diversity than the 16S rRNA gene, including rplF (encoding 50S ribosomal protein L6), $\arg F$ (ornithine carbamoyltransferase), recA (DNA recombination/repair protein RecA), rpoB (DNA-directed RNA polymerase subunit beta), rpoD (RNA polymerase sigma factor RpoD), polI (DNAdirected DNA polymerase I), ribII (ribonuclease II), aspS (aspartate-tRNA ligase), gyrB (DNA gyrase subunit B) and porin precursor (por) exist in all Neisseria species (Volokhov et al. 2018). As in previous studies, similarities for these genes between strain $10023^{\mathrm{T}}$ and closely related Neisseria type strains were analysed.

Chemotaxonomic analyses

Test and reference strains were cultured on BHI agar with $5 \%$ defibrinated sheep blood plate at $37{ }^{\circ} \mathrm{C}, 5 \%$ $\mathrm{CO}_{2}$, and harvested in the late-exponential growth phase. To determine fatty acid content and 
compositions, five solutions were prepared in advance (solution I: $45 \mathrm{~g} \mathrm{NaOH}$ in $1: 1$ distilled water/ methanol; solution II: $190 \mathrm{~mL}$ concentrated hydrochloric acid/275 mL methanol in $135 \mathrm{~mL}$ distilled water; solution III: 1:1 n-hexane/MTBE; solution IV: $10.8 \mathrm{~g} \mathrm{NaOH}$ in $900 \mathrm{~mL}$ distilled water; solution V: saturated sodium chloride). The cellular fatty acids of test and reference strains were saponified, methylated and extracted according to the protocol of the Sherlock Microbial Identification System (MIDI) and analysed by GC (model 6890, Hewlett Packard).

Morphological, physiological and biochemical analysis

Type strain $10023^{\mathrm{T}}$ was cultivated for 2 days at $37{ }^{\circ} \mathrm{C}$ on BHI agar with $5 \%$ defibrinated sheep blood for morphological observation using a transmission electron microscope (HT-7700). Gram-staining was performed using the Color Gram 2 kit (bioMérieux, France), according to the manufacturer's protocol. Motility was examined by inoculation in a semisolid $\mathrm{BHI}$ broth. The temperature range for growth was determined using BHI agar with 5\% defibrinated sheep blood for a week of incubation at $4-45{ }^{\circ} \mathrm{C}$. Salt tolerance was tested in BHI broth supplemented with $0-8.0 \%$ (w/v) $\mathrm{NaCl}$ (at $1.0 \%$ intervals) after 5 days of incubation at $37{ }^{\circ} \mathrm{C}$. The $\mathrm{pH}$ range $(\mathrm{pH} 3.0-11.0$, at 1 $\mathrm{pH}$ unit intervals) for growth was determined using BHI broth that was buffered with citrate/phosphate buffer or Tris/hydro-chloride buffer (Wang et al. 2015). Anaerobic growth was tested on BHI agar with $5 \%$ defibrinated sheep blood plate using the Anaerobic workstation (ELECTROTEK, Britain), in which the air was substituted with $90 \% \mathrm{~N}_{2}, 5 \% \mathrm{H}_{2}$ and $5 \% \mathrm{CO}_{2}$. Bacteroides vulgatus DSM $1447^{\mathrm{T}}$ was used as a positive control. Growth on different media was detected, including nutrient agar, BHI, Columbia blood or chocolate plates and MacConkey agar. Phenotypic characteristics (API NH, API ZYM) were tested strictly based on the manufacturers' instructions with cells cultured on BHI agar with $5 \%$ defibrinated sheep blood under the conditions of $37{ }^{\circ} \mathrm{C}$ and $5 \%$ $\mathrm{CO}_{2}$. An oxidase activity test was performed using a commercial dropper oxidase reagent (Becton-Dickinson, USA). Utilisation of Simmons' citrate was tested using Simmons citrate agar.

\section{Results and discussion}

Phylogenetic analysis

The sequences of the 16S rRNA genes of isolates $10023^{\mathrm{T}}$ and 10010 were found to be identical. Sequence analysis indicated these two novel strains to be closely related to Neisseria zalophi CSL $7565^{\mathrm{T}}$, N. wadsworthii WC $05-9715^{\mathrm{T}}$ and $N$. canis ATCC $14687^{\mathrm{T}}$ with similarities of $96.98,96.92$ and $96.79 \%$, respectively. Phylogenetic trees constructed using different methods based on 16S rRNA genes were quite similar (Neighbour-Joining tree for Fig. 1 and Maximum-Likelihood tree for Supplementary Fig. S1). These two novel strains grouped with members of the genus Neisseria and reside in an independent clade that harbours 7 Neisseria species, including N. zalophi, N. wadsworthii, N. canis, Neisseria zoodegmatis, Neisseria dentiae, Neisseria shayeganii and Neisseria dumasiana.

Despite significant 16S rRNA gene sequence difference between these two novel strains and their relatives, to precisely clarify the taxonomic position, phylogenetic analysis based on the $r p l F$ gene was performed, which showed that these two novel isolates reside in a well-supported branch and can be easily discriminated from phylogenetically closely related species (Fig. 2).

Average nucleotide identity and genes similarity analysis

The ANI values between strain $10023^{\mathrm{T}}$ and other species in the genus Neisseria ranged from 73.5$79.3 \%$ (Table S1), lower than the 95\% threshold, which supports the conclusion that these two isolates belong to a novel species. Moreover, the ANI value between isolates $10023^{\mathrm{T}}$ and 10010 was $99.7 \%$, further proving they belong to the same species.

The circular chromosome of $10023^{\mathrm{T}}$ is $2,496,444 \mathrm{bp}$ in size with an overall $\mathrm{G}+\mathrm{C}$ content of $54.0 \mathrm{~mol} \%$. The genome of $10023^{\mathrm{T}}$ encompasses 2332 protein coding sequences, 58 tRNAs and 12 rRNAs, with four copies each of the $16 \mathrm{~S}, 23 \mathrm{~S}$ and $5 \mathrm{~S}$ rRNA genes. As predicted by the virulence factor database VFDB (http://www.mgc.ac.cn/) (Chen et al. 2016) and verified by Blast searches at NCBI, the genome of type strain $10023^{\mathrm{T}}$ possesses typical Neisseria genes, including genes encoding putative 


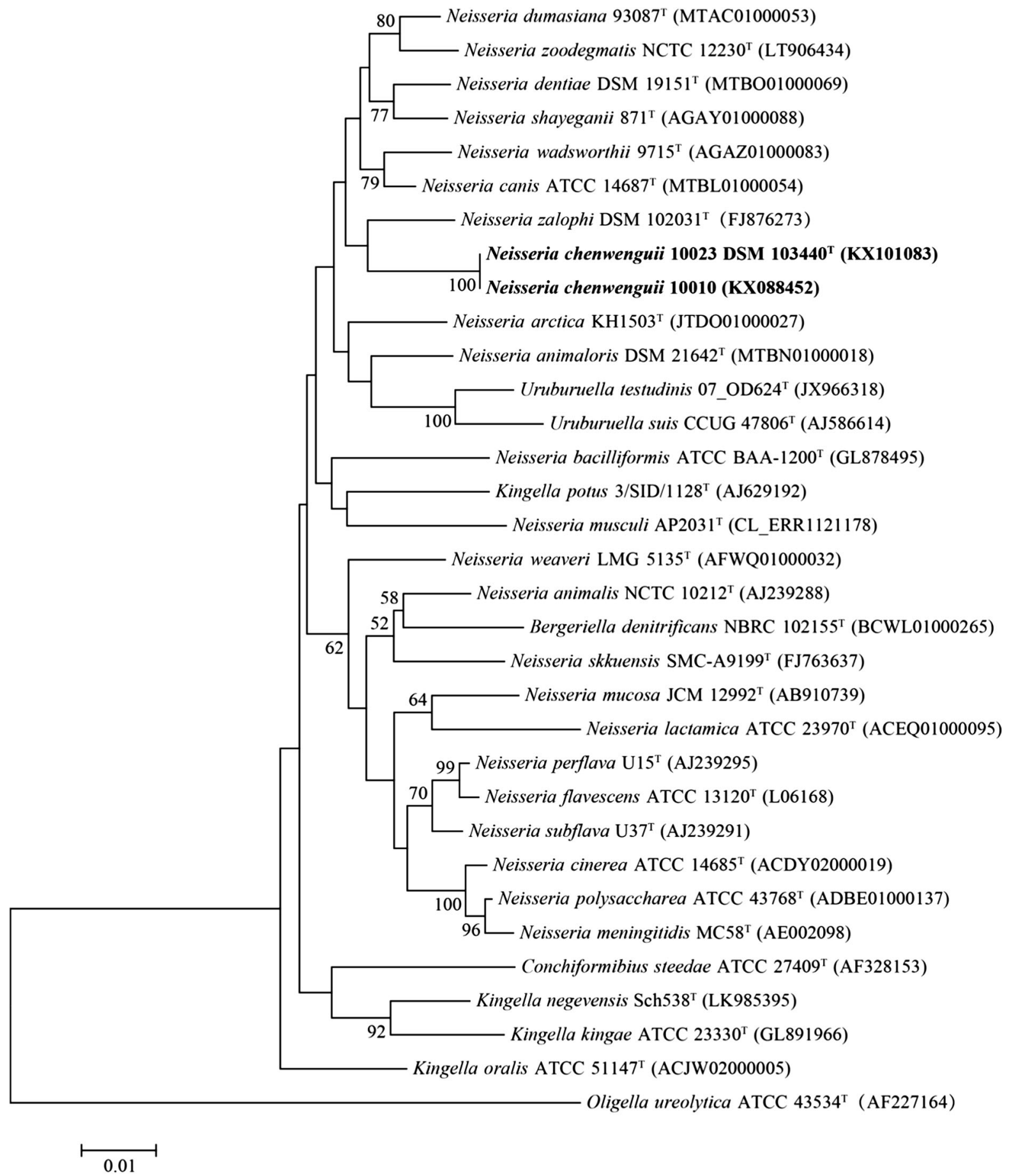

Fig. 1 Neighbor-joining tree reconstructed from 16S rRNA gene sequences of Neisseria chenwenguii sp. nov. and the 30 closest taxa returned from a BLAST search of the EzTaxon database. The tree was constructed based on the Kimura 2-parameter model conducted in MEGA7.0 rooted with Oligella ureolytica DSM $18253^{\mathrm{T}}$. The percentage of replicate trees in which the associated taxa clustered together in the bootstrap test (1000 replicates) are shown next to the branches. The tree is drawn to scale, with branch lengths in the same units as those of the evolutionary distances used to infer the phylogenetic tree. There were a total of 1294 positions in the final dataset. Bar, 0.01 expected changes per site 


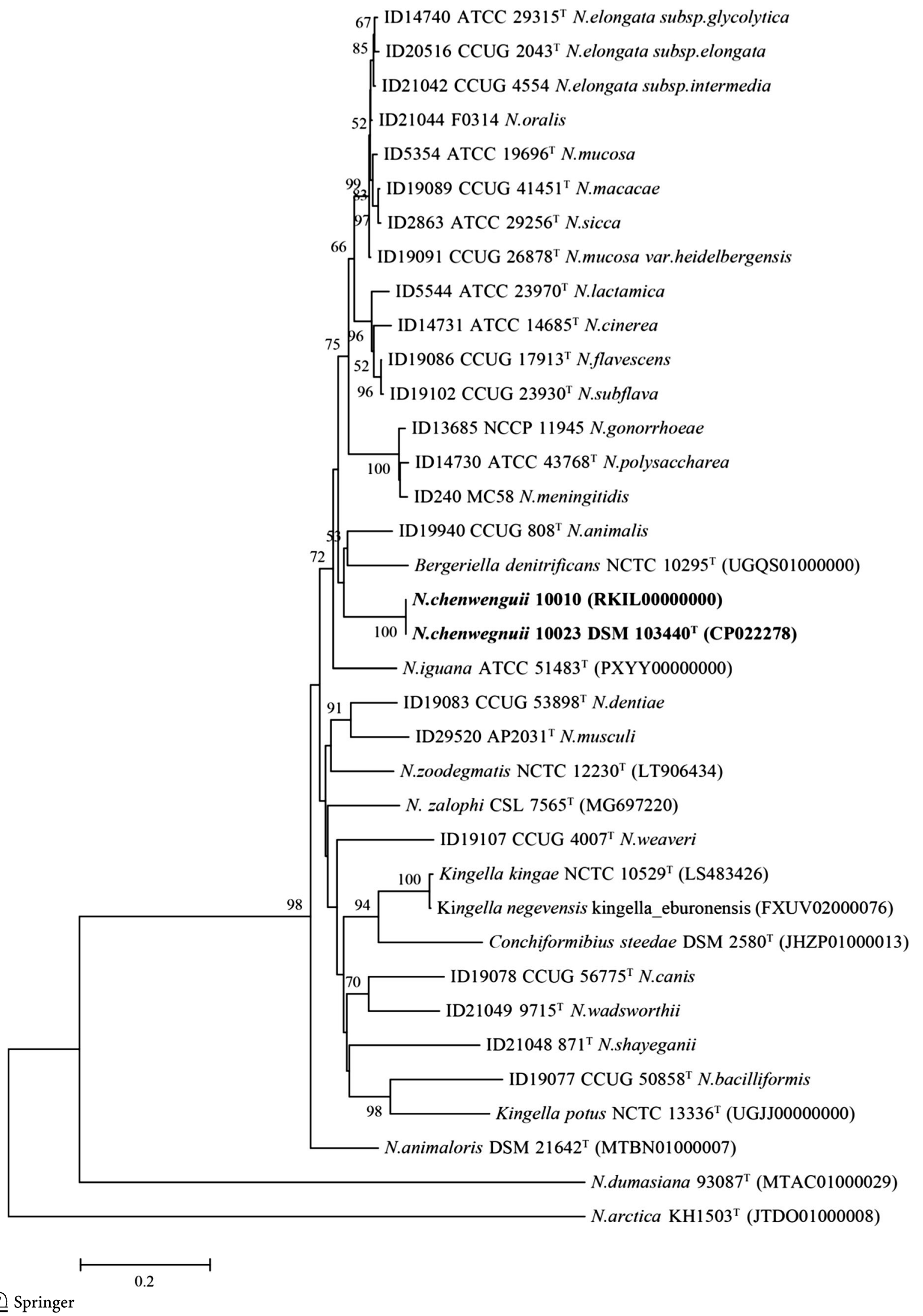


४Fig. 2 Neighbor-Joining phylogenetic tree based on $r p l F$ gene for the two isolates of Neisseria chenwenguii sp. nov. and 34 additional taxa with a total of 468 positions in the final dataset. The percentage of replicate trees in which the associated taxa clustered together in the bootstrap test (1000 replicates) are shown next to the branches. The tree is drawn to scale, with branch lengths in the same units as those of the evolutionary distances used to infer the phylogenetic tree. The evolutionary distances were computed using the Maximum Composite Likelihood method and are in the units of the number of base substitutions per site

virulence-associated factors, such as CapC, catalase, lipopolysaccharide heptosyltransferase II, lipopolysaccharide heptosyltransferase I, LptA and LptB. The similarities of these virulence-associated factors between isolate $10023^{\mathrm{T}}$ and other Neisseria spp were in the range of $53.0-61.3 \%, 81.9-90.8 \%$, $78.6-83.9 \%, \quad 71.8-77.1 \%, \quad 61.3-73.6 \%$ and $84.3-87.7 \%$, respectively.

The genes similarities of $r p l F$, gyrB, rpoB, recA, $\arg F$, rpoD, polI, ribII, aspS and por porin precursor between the type strain $10023^{\mathrm{T}}$ and its closely related species (N. zalophi CSL $7565^{\mathrm{T}}, N$. wadsworthii $9715^{\mathrm{T}}$ and $N$. canis ATCC $14687^{\mathrm{T}}$ ) ranged from 73.0 to $86.4 \%$ (Table 1), which clearly demonstrates that isolate $10023^{\mathrm{T}}$ is different from its relatives. The similarities of the $r p l F, g y r B, r p o B, r e c A, \arg F, r p o D$, polI, ribII, aspS and por sequences between strains $10023^{\mathrm{T}}$ and 10010 are $100.0 \%, 99.8 \%, 100.0 \%$, $99.9 \%, 98.5 \%, 99.9 \%, 99.9 \%, 100.0 \%, 99.9 \%$ and $100.0 \%$, respectively.

The DNA uptake sequence (DUS), a highly repetitive genomic element, plays important roles in the process of DNA transformation that ensures that all Neisseria species are competent. A 12-base sequence termed agDUS is substantial in the genomes of Neisseria subflava, Neisseria flavescens, Neisseria mucosa, Neisseria bacilliformis and Neisseria weaver
(Frye et al. 2013; Mell and Redfield 2014). There are over 4000 copies of the agDUS in the $10023^{\mathrm{T}}$ genome as analysed by WebLogo and EMBOSS (Crooks et al. 2004; Rice et al. 2000). This also supports the conclusion that the novel species is a member of the genus Neisseria.

\section{Chemotaxonomic characterisation}

$\mathrm{C}_{16: 0}$ and summed feature $3\left(\mathrm{C}_{16: 1 \omega 7 \mathrm{c}} / \mathrm{C}_{16: 1 \omega 6 \mathrm{c}}\right)$ were identified as the predominant fatty acids in strains $10023^{\mathrm{T}}$ and 10010 , representing about $31.6 \% / 29.5 \%$ and $34.9 \% / 29.7 \%$ of the total fatty acids, respectively (Table 2). The major cellular fatty acids present in $N$. zalophi, N. wadsworthii and $N$. canis were $\mathrm{C}_{16: 0} /$ $\mathrm{C}_{16: 1 \omega 9 \mathrm{c}}, \mathrm{C}_{16: 0} / \mathrm{C}_{18: 1 \omega 7 \mathrm{c}} /$ summed feature $3\left(\mathrm{C}_{16: 1 \omega 7 \mathrm{c}} /\right.$ $\left.\mathrm{C}_{16: 1 \omega 6 \mathrm{c}}\right)$ and $\mathrm{C}_{16: 0} / \mathrm{C}_{18: 1 \omega 7 \mathrm{c}}$, respectively. Hence, these two novel isolates can be differentiated from their close relatives by their unique fatty acids profiles (Table 2).

Morphological, physiological and biochemical analysis

Cells were observed to be Gram-negative, non-motile, piliated coccoid without flagella (Supplementary Fig. S2), in single, pairs, or chains, 0.2-0.9 um in diameter under transmission electron microscope. Growth occurs at $22-40{ }^{\circ} \mathrm{C}$ (optimum at $37{ }^{\circ} \mathrm{C}$ ) on BHI agar with $5 \%$ defibrinated sheep blood. Growth was also observed on nutrient agar, BHI, Columbia blood agar or chocolate plates, but not on MacConkey agar after 7 days. Further, cells were found to grow in the presence of $0-4 \%(\mathrm{w} / \mathrm{v}) \mathrm{NaCl}$ (optimum in $1 \%(\mathrm{w} /$ v) $\mathrm{NaCl}$ ) and at a $\mathrm{pH}$ range of 6.0-8.0 (optimum at $\mathrm{pH}$ 7.0). These two novel isolates can grow under anaerobic conditions and are negative for utilisation of Simmon's citrate. The cells produce catalase and

Table 1 Similarities (\%) for the $r p l F$, gyrB, rpoB, recA, $\arg F$, rpoD, polI, ribII, aspS and porin precursor genes between Neisseria chenwenguii $10023^{\mathrm{T}}$ and closely related Neisseria-type strains

\begin{tabular}{|c|c|c|c|c|c|c|c|c|c|c|}
\hline \multirow[t]{2}{*}{ Strain } & \multicolumn{10}{|c|}{ Similarity $(\%)$ with type strain $10023^{\mathrm{T}}$} \\
\hline & $r p l F$ & gyrB & гров & recA & $\arg F$ & rpoD & polI & ribII & $\operatorname{aspS}$ & porin precursor \\
\hline Neisseria zalophi CSL $7565^{\mathrm{T}}$ & 80.0 & 77.9 & 79.1 & 81.5 & 73.6 & 78.7 & 78.5 & 74.6 & 84.2 & 70.6 \\
\hline Neisseria wadsworthii $9715^{\mathrm{T}}$ & 77.6 & 75.7 & 79.2 & 80.1 & 78.7 & 76.6 & 82.7 & 73.0 & 85.8 & 71.7 \\
\hline Neisseria canis ATCC $14687^{\mathrm{T}}$ & 75.0 & 78.2 & 80.7 & 82.5 & 78.1 & 81.0 & 83.0 & 73.1 & 86.4 & 70.0 \\
\hline
\end{tabular}


Table 2 The cellular fatty acid profiles (\%) of Neisseria chenwenguii sp. nov. and its relatives

\begin{tabular}{|c|c|c|c|c|c|}
\hline Fatty acid & 1 & 2 & 3 & 4 & 5 \\
\hline $\mathrm{C}_{12: 0}$ & 6.6 & 9.5 & 0.6 & 6.2 & 8.9 \\
\hline $\mathrm{C}_{14: 0}$ & 4.1 & 3.7 & 0.7 & 2.5 & 2.2 \\
\hline $\mathrm{C}_{16: 0}$ & 31.6 & 29.5 & 39.2 & 29.4 & 36.2 \\
\hline $\mathrm{C}_{18: 0}$ & 1.1 & 1.9 & 1.0 & 1.6 & 3.1 \\
\hline $\mathrm{C}_{16: 1} \omega 9 \mathrm{c}$ & ND & ND & 38.8 & ND & ND \\
\hline $\mathrm{C}_{18: 1} \omega 7 \mathrm{c}$ & 6.8 & 7.2 & 0.3 & 26.1 & 23.9 \\
\hline $\mathrm{C}_{18: 1} \omega 9 \mathrm{c}$ & 2.2 & 1.9 & 14.9 & 1.5 & 2.0 \\
\hline Summed feature $2^{\mathrm{a}}$ & 9.6 & 12.1 & ND & 2.7 & 4.7 \\
\hline Summed feature $3^{\mathrm{a}}$ & 34.9 & 29.7 & ND & 23.1 & 7.6 \\
\hline
\end{tabular}

Strains: $1,10023^{\mathrm{T}} ; 2,10010 ; 3$, Neisseria zalophi CSL $7565^{\mathrm{T}}$ (Volokhov et al. 2018); 4, Neisseria wadsworthii WC05$9715^{\mathrm{T}} ;$ 5, Neisseria canis ATCC $14687^{\mathrm{T}}$. Values are percentages of total fatty acids. The two or three most abundant fatty acids for each isolate are in bold

$N D$ not detected

${ }^{a}$ Summed features consist of groups of two or three fatty acids that cannot be separated using the MIDI System. Summed feature 2 is composed of $\mathrm{C}_{14: 0} 3_{3}-\mathrm{OH} /$ isol- $\mathrm{C}_{16: 1}$; summed feature 3 is composed of $C_{16: 1} \omega 7 \mathrm{c} / C_{16: 1} \omega 6 \mathrm{c}$ cannot reduce nitrate to nitrite. Strain 10010 was found to be positive for esterase (C4), esterase lipase (C8), naphthol-AS-BI-phosphohydrolase, proline arylamidase and $\alpha$-galactosidase, which discriminates this strain from the type strain $10023^{\mathrm{T}}$ (Table 3).

Some key biochemical features of the novel species are helpful in distinguishing it from closely related taxa, which can be summarised as follows: (i) the novel species is positive for urea utilisation and cannot reduce nitrate to nitrite, which differentiates it from $N$. zalophi, N. wadsworthii and N. canis; (ii) according to the reaction patterns using API NH, the novel species can ferment fructose and maltose, and is ureasepositive; (iii) the novel species can be distinguished from closely related species based on the reaction of alkaline phosphatase and $\beta$-galactosidase (positive for these two novel isolates) using API ZYM.

However, when characterising potential new species to the genus Neisseria, membership of closely related genera should be excluded. The presence of catalase activity and the absence of motility observed
Table 3 Characteristics that differentiate Neisseria chenwenguii sp. nov. from closely related Neisseria species
Strains: $1,10023^{\mathrm{T}} ; 2$,

10010; 3, Neisseria zalophi

CSL $7565^{\mathrm{T}}$ (Volokhov et al.

2018); 4, Neisseria wadsworthii WC05-9715 ${ }^{\mathrm{T}}$;

5, Neisseria canis ATCC $14687^{\mathrm{T}}$

+ positive, - negative

\begin{tabular}{|c|c|c|c|c|c|}
\hline Characteristic & 1 & 2 & 3 & 4 & 5 \\
\hline Urea & + & + & - & - & - \\
\hline Nitrate & - & - & + & + & + \\
\hline $\mathrm{pH}$ range & $6-8$ & $6-8$ & ND & $6-10$ & $6-10$ \\
\hline Growth in presence of $\mathrm{NaCl}(\%)$ & 4 & 4 & 6 & 2 & 2 \\
\hline Anaerobic growth & + & + & - & + & + \\
\hline \multicolumn{6}{|l|}{ API ZYM } \\
\hline Alkaline phosphatase & + & + & - & - & - \\
\hline Esterase (C4) & - & + & + & + & + \\
\hline Esterase lipase (C8) & - & + & + & - & + \\
\hline Valine arylamidase & - & - & + & - & - \\
\hline Cystine arylamidase & - & - & + & - & - \\
\hline Acid phosphatase & + & + & + & - & - \\
\hline Naphthol-AS-BIphosphohydrolase & - & + & + & + & + \\
\hline$\alpha$-Galactosidase & - & + & - & - & - \\
\hline$\beta$-Galactosidase & + & + & - & - & - \\
\hline \multicolumn{6}{|l|}{ API NH } \\
\hline Glucose & + & + & - & + & + \\
\hline Fructose & + & + & - & + & - \\
\hline Maltose & + & + & - & + & - \\
\hline Sucrose & + & + & - & + & + \\
\hline Urease & + & + & - & - & - \\
\hline Proline arylamidase & - & + & + & + & + \\
\hline$\gamma$-Glutamyltransferase & + & + & - & + & - \\
\hline
\end{tabular}


in these two novel isolates exclude membership in the genera Bergeriella, Eikenella and Kingella, which are catalase-negative. Our phylogenetic analysis based on the genes for $16 \mathrm{~S}$ rRNA and rplF, and ANI analysis, showed that the novel species can be easily discriminated from all phylogenetically close relatives and other species in genus Neisseria.

Taken together, results from biochemical tests, fatty acid analyses, ANI and phylogenetic analyses based on the 16S rRNA and $r p l F$ genes all support the conclusion that isolates $10023^{\mathrm{T}}$ and 10010 belong to the genus Neisseria and deserve classification as a novel species within the genus, for which the name Neisseria chenwenguii sp. nov. is proposed, with strain $10023^{\mathrm{T}}$ as the type strain.

Description of Neisseria chenwenguii sp. nov.

Neisseria chenwenguii (chen.wen.gui'i. N.L. gen. n. chenwenguii in honour of Professor Wengui Chen, an academician who studied acute infectious disease prevention and control in the 1950s in China).

Strains are moist, small, greyish, convex with regular margins, $0.6-1.0 \mathrm{~mm}$ in diameter after $48 \mathrm{~h}$ of growth at $37{ }^{\circ} \mathrm{C}$ under $5 \% \mathrm{CO}_{2}$ on BHI agar with $5 \%$ defibrinated sheep blood. Optimal temperature is $37{ }^{\circ} \mathrm{C}$. Negative for Simmons' citrate utilisation and indole production, and positive for urea utilisation. In the API ZYM system, positive for alkaline phosphatase, leucine arylamidase, acid phosphatase and $\beta$ galactosidase. In the API NH gallery can produce acid from glucose, fructose, maltose and sucrose, and is positive for urease and $\gamma$-glutamyltransferase. The main cellular fatty acids are $C_{16: 0}$ and $C_{16: 1 \omega 7 \mathrm{c}}$ $\mathrm{C}_{16: 1 \omega 6 \mathrm{c}}$. The $\mathrm{G}+\mathrm{C}$ content of the type strain is $54.0 \mathrm{~mol} \%$.

The type strain, $10023^{\mathrm{T}}$ (= DSM $103440^{\mathrm{T}-}$ $=$ CGMCC $1.15736^{\mathrm{T}}$ ), was isolated from the rectal content of a wild plateau pika (Ochotona curzoniae) on the Qinghai-Tibet Plateau, China. The GenBank accession numbers for the 16S rRNA gene sequences of strains $10023^{\mathrm{T}}$ and 10010 are KX088452 and KX101083, respectively. The GenBank/EMBL/DDBJ accession numbers for the genome sequences of isolates $10023^{\mathrm{T}}$ and 10010 are CP022278 and RKIL00000000, respectively.
Authors' contributions GZ analysed most of the data, and wrote the initial draft of the paper. JY, XHL contributed to providing critical revisions to this article. SL, DJ, JP, XB, XL and YX were responsible for collecting samples. $\mathrm{YH}$ contributed to take electron microscope photographs. CC contributed to carrying out additional analyses. JX conceived of the study and designed the study. All authors discussed the results and revised the manuscript.

Funding This study was funded by National Key R\&D Program of China (2016YFC1201903), National Natural Science Foundation of China (81290340 and 81290345) and Sanming Project of Medicine in Shenzhen (SZSM201811071).

\section{Compliance with ethical standards}

Conflict of interest The authors declare that they have no conflict of interest.

Ethical statement All procedures performed in studies involving animals were in accordance with the ethical standards of the institution or practice at which the studies were conducted.

Informed consent Informed consent was obtained from all individual participants included in the study.

Open Access This article is distributed under the terms of the Creative Commons Attribution 4.0 International License (http:// creativecommons.org/licenses/by/4.0/), which permits unrestricted use, distribution, and reproduction in any medium, provided you give appropriate credit to the original author(s) and the source, provide a link to the Creative Commons license, and indicate if changes were made.

\section{References}

Adeolu M, Gupta RS (2013) Phylogenomics and molecular signatures for the order Neisseriales: proposal for division of the order Neisseriales into the emended family Neisseriaceae and Chromobacteriaceae fam. nov. Antonie Van Leeuwenhoek 104:1-24

Bai X, Xiong Y, Lu S, Jin D, Lai X, Yang J, Niu L, Hu S, Meng $\mathrm{X}, \mathrm{Pu}$ J, Ye C, Xu J (2016) Streptococcus pantholopis sp. nov., isolated from faeces of the Tibetan antelope (Pantholops hodgsonii). Int $\mathrm{J}$ Syst Evol Microbiol 66:3281-3286. https://doi.org/10.1099/ijsem.0.001189

Bennett JS, Jolley KA, Maiden MC (2013) Genome sequence analyses show that Neisseria oralis is the same species as 'Neisseria mucosa var. heidelbergensis'. Int J Syst Evol Microbiol 63:3920-3926. https://doi.org/10.1099/ijs.0. 052431-0

Bennett JS, Watkins ER, Jolley KA, Harrison OB, Maiden MC (2014) Identifying Neisseria species by use of the 50S ribosomal protein L6 $(r p l F)$ gene. J Clin Microbiol 52:1375-1381. https://doi.org/10.1128/JCM.03529-13

Berlin K, Koren S, Chin CS, Drake JP, Landolin JM, Phillippy AM (2015) Assembling large genomes with single- 
molecule sequencing and locality-sensitive hashing. Nat Biotechnol 33:623-630. https://doi.org/10.1038/nbt.3238

Chen L, Zheng D, Liu B, Yang J, Jin Q (2016) VFDB 2016: hierarchical and refined dataset for big data analysis10 years on. Nucleic Acids Res 44:D694-D697. https:// doi.org/10.1093/nar/gkv1239

Crooks GE, Hon G, Chandonia JM, Brenner SE (2004) WebLogo: a sequence logo generator. Genome Res 14:1188-1190. https://doi.org/10.1101/gr.849004

Frye SA, Nilsen M, Tonjum T, Ambur OH (2013) Dialects of the DNA uptake sequence in Neisseriaceae. PLoS Genet 9:e1003458. https://doi.org/10.1371/journal.pgen.1003458

Hansen CM, Himschoot EA, Hare RF, Meixell BW, Hemert CV, Hueffer K (2017) Neisseria arctica sp. nov., isolated from nonviable eggs of greater white-fronted geese (Anser albifrons) in Arctic Alaska. Int J Syst Evol Microbiol 67:1115-1119. https://doi.org/10.1099/ijsem.0.001773

Hu S, Jin D, Lu S, Liu S, Zhang J, Wang Y, Bai X, Xiong Y, Huang Y, Xu H, Wang Y, Du X, Ye C, Hänninen ML, Xu J (2015) Helicobacter himalayensis sp. nov. isolated from gastric mucosa of Marmota himalayana. Int J Syst Evol Microbiol 65:1719-1725. https://doi.org/10.1099/ijs.0. 000163

Kumar S, Stecher G, Tamura K (2016) MEGA7: molecular evolutionary genetics analysis version 7.0 for bigger datasets. Mol Biol Evol 33:1870-1874. https://doi.org/10. 1093/molbev/msw054

Liu S, Jin D, Lan R, Wang Y, Meng Q, Dai H, Lu S, Hu S, Xu J (2015) Escherichia marmotae sp. nov., isolated from faeces of Marmota himalayana. Int J Syst Evol Microbiol 65:2130-2134. https://doi.org/10.1099/ijs.0.000228

Mell JC, Redfield RJ (2014) Natural competence and the evolution of DNA uptake specificity. $\mathrm{J}$ Bacteriol 196:1471-1483. https://doi.org/10.1128/JB.01293-13

Meng X, Lu S, Lai XH, Wang Y, Wen Y, Jin D, Yang J, Xu J (2017a) Actinomyces liubingyangii sp. nov. isolated from the vulture Gypaetus barbatus. Int J Syst Evol Microbiol 67:1873-1879. https://doi.org/10.1099/ijsem.0.001884

Meng X, Lu S, Wang Y, Lai XH, Wen Y, Jin D, Yang J, Bai X, Zhang G, Pu J, Lan R, Xu J (2017b) Actinomyces vulturis sp. nov., isolated from Gyps himalayensis. Int J Syst Evol Microbiol 67:1720-1726. https://doi.org/10.1099/ijsem.0. 001851

Murphy J, Devane ML, Robson B, Gilpin BJ (2005) Genotypic characterization of bacteria cultured from duck faeces. J Appl Microbiol 99:301-309. https://doi.org/10.1111/j. 1365-2672.2005.02590.x

Neilan BA, Wilton AN, Jacobs D (1997) A universal procedure for primer labelling of amplicons. Nucleic Acids Res 25:2938-2939

Niu L, Lu S, Hu S, Jin D, Lai X, Yang J, Chen C, Wang Y, Bai X, Lan R, Lv G, Xie Y, Ye C, Xu J (2016) Streptococcus halotolerans sp. nov. isolated from the respiratory tract of Marmota himalayana in Qinghai-Tibet Plateau of China. Int J Syst Evol Microbiol 66:4211-4217. https://doi.org/10. 1099/ijsem.0.001337

Niu L, Lu S, Lai XH, Hu S, Chen C, Zhang G, Yang J, Jin D, Wang Y, Lan R, Lu G, Xie Y, Ye C, Xu J (2017) Streptococcus himalayensis sp. nov., isolated from the respiratory tract of Marmota himalayana. Int J Syst Evol Microbiol 67:256-261. https://doi.org/10.1099/ijsem.0. 001609

Rice P, Longden I, Bleasby A (2000) EMBOSS: the European Molecular Biology Open Software Suite. Trends Genet 16:276-277

Richter M, Rossello-Mora R (2009) Shifting the genomic gold standard for the prokaryotic species definition. Proc Natl Acad Sci USA 106:19126-19131. https://doi.org/10.1073/ pnas.0906412106

Thompson JD, Gibson TJ, Higgins DG (2002) Multiple sequence alignment using ClustalW and ClustalX. Curr Protoc Bioinformatics Chapter 2:Unit 2.3. https://doi.org/ 10.1002/0471250953.bi0203s00

Virji M (2009) Pathogenic neisseriae: surface modulation, pathogenesis and infection control. Nat Rev Microbiol 7:274-286. https://doi.org/10.1038/nrmicro2097

Volokhov DV, Amselle M, Bodeis-Jones S, Delmonte P, Zhang S, Davidson MK, Gulland FM, Chizhikov VE (2018) Neisseria zalophi sp. nov., isolated from oral cavity of California sea lions (Zalophus californianus). Arch Microbiol 200:819-828. https://doi.org/10.1007/s00203018-1499-x

Wang X, Yang HX, Zhang YK, Zhu SJ, Liu XW, Zhang H, Zhang CF, Zhao CR, Hu G, Hong Q (2015) Luteimonas soli sp. nov., isolated from farmland soil. Int J Syst Evol Microbiol 65:4809-4815. https://doi.org/10.1099/ijsem.0. 000652

Weyand NJ, Ma M, Phifer-Rixey M, Taku NA, Rendón MA, Hockenberry AM, Kim WJ, Agellon AB, Biais N, Suzuki TA, Goodyer-Sait L, Harrison OB, Bratcher HB, Nachman MW, Maiden MC, So M (2016) Isolation and characterization of Neisseria musculi sp. nov., from the wild house mouse. Int J Syst Evol Microbiol 66:3585-3593. https:// doi.org/10.1099/ijsem.0.001237

Wolfgang WJ, Carpenter AN, Cole JA, Gronow S, Habura A, Jose S, Nazarian EJ, Kohlerschmidt DJ, Limberger R, Schoonmaker-Bopp D, Spröer C, Musser KA (2011) Neisseria wadsworthii sp. nov. and Neisseria shayeganii sp. nov., isolated from clinical specimens. Int J Syst Evol Microbiol 61:91-98. https://doi.org/10.1099/ijs.0.0224260

Wolfgang WJ, Passaretti TV, Jose R, Cole J, Coorevits A, Carpenter AN, Jose S, Van Landschoot A, Izard J, Kohlerschmidt DJ, Vandamme P, Dewhirst FE, Fisher MA, Musser KA (2013) Neisseria oralis sp. nov., isolated from healthy gingival plaque and clinical samples. Int $\mathbf{J}$ Syst Evol Microbiol 63:1323-1328. https://doi.org/10. 1099/ijs.0.041731-0

Wroblewski D, Cole J, McGinnis J, Perez M, Wilson H, Mingle LA, Musser KA, Wolfgang WJ (2017) Neisseria dumasiana sp. nov. from human sputum and a dog's mouth. Int J Syst Evol Microbiol 67:4304-4310. https://doi.org/10. 1099/ijsem.0.002148

Publisher's Note Springer Nature remains neutral with regard to jurisdictional claims in published maps and institutional affiliations. 